Revista de Derecho

\title{
Derecho cultural y amparo del coleo en el marco de la protección animal en el ordenamiento jurídico colombiano
}

Cultural law and revision under the defense of coleus in the framework of animal protection in the Colombian legal system

\author{
Tirson Mauricio Duarte Molina \\ Investigador en Derecho en Universidad de San Buenaventura, Colombia \\ tirsonmduarte@gmail.com \\ ORCID: 0000-0002-6240-2609
}

DOI: https://doi.org/10.32719/26312484.2020.34.9

Fecha de recepción: 28 de octubre de 2019

Fecha de aceptación: 15 de enero de 2020

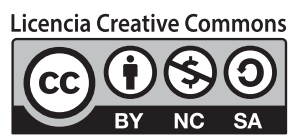




\section{RESUMEN}

El reconocimiento de manifestaciones culturales en el ámbito jurídico colombiano se hace maleable al identificar, proteger y garantizar los derechos que sobre ella versen. La doctrina por su parte ha identificado los principios y las características propias de estas manifestaciones, sin encontrar una posición unificada respecto de la forma de construcción de la cultura desde el individuo o su participación en el colectivo que lo rodea. Esto ha generado que el ordenamiento colombiano no considere un criterio unificado sobre la noción de cultura y sobre el derecho cultural. De esta manera, ante las preguntas orientadoras ¿qué es cultura? y ¿qué debe considerarse como una manifestación cultural?, se ha hecho necesario establecer una delimitación de estas categorías con el fin de comprender cuándo una práctica debe o no ser cobijada por el derecho cultural, usando el test de culturalidad como método para la determinación de prácticas como manifestaciones culturales. Para el caso concreto, la práctica conocida como "coleo", en la que se da una divergencia entre derecho cultural y protección animal, se realiza una caracterización y análisis a la luz de esta protección a la fauna y de los principios que fundamentan la protección cultural realizando una validación de su esencia cultural.

PALABRAS ClAVE: derecho cultural, coleo, protección animal, manifestación cultural, identidad cultural, test de culturalidad.

\section{ABSTRACT}

The recognition of cultural manifestations in the Colombian jurisdiction becomes malleable when it comes to identifying, protecting and guaranteeing the rights related. The doctrine has identified the principles and inner characteristics of these manifestations, without finding a unified position regarding the way of building the culture from the individual or their participation in the surrounded collective. The above has generated a Colombian system where it is not considered itself a unified criterion on the notion of culture and the cultural right by which it is protected, as well as the prerogatives that must be given before its concession. Thus, before the guiding questions, what is culture? And what should be considered as a cultural manifestation? It has become necessary to establish a boundary of these categories in order to understand objectively when a practice should or should not be covered by cultural law; and for this, it's used the culturality test as a method for determining practices as cultural manifestations. For a concrete case, the practice known as "coleo" appears, where there is a divergence between cultural law and animal protection, a characterization and analysis is carried out in the light of this protection of wildlife and the principles that underlie cultural protection as a validation of their cultural essence.

KEYWORDS: cultural law, coleus, animal protection, cultural manifestation, cultural identity, culturality test. 


\title{
INTRODUCCIÓN
}

\begin{abstract}
A 1 realizar un rastreo en el ordenamiento colombiano de lo que es el derecho cultural, no se logra encontrar una acepción clara; realmente han sido pocos los pronunciamientos legales, judiciales y doctrinales sobre el contenido de este derecho. De la misma manera ocurre cuando, desde el derecho, se pregunta por lo que es y se debe considerar como cultura. De allí que ha sido importante para algunos autores establecer un concepto de cultura y las prácticas que deben considerarse como tales, para posteriormente lograr la caracterización del derecho cultural, siendo este último su principal fin.
\end{abstract}

La garantía de protección por parte del Estado, al tener una delimitación del derecho insuficiente, podría verse subsanada en la medida en que, al darse tal caracterización a través de una evaluación de los elementos que componen cada práctica individualmente apreciada, se puede estimar el alcance de la protección del derecho, fenómeno por el cual la doctrina ha identificado criterios de culturalidad que han sido sistematizados en el llamado test de culturalidad en beneficio de la identificación y protección de manifestaciones.

Así, a través de la sistematización de conceptos filosóficos, doctrinales y referencias a pronunciamientos del máximo tribunal de Colombia, la Corte Constitucional, se estudia la teoría cultural teniendo en cuenta autores contemporáneos relacionados con la identidad cultural y el multiculturalismo como Kymlicka, Jameson, Žižek, Real de Alcalá, y Villoro; estudio dentro del cual se dará un análisis a la perspectiva individualista y colectiva de la noción de cultura, análisis realizado de manera previa por Duarte.

En Colombia se ha dado una protección extensiva a diferentes manifestaciones y prácticas desde la Constitución Política de 1991, principalmente las étnicas. No obstante, algunas de ellas han sido generadas por la vida en comunidad o han migrado desde diferentes latitudes, motivos por los cuales no se ha extendido la protección a ellas más que a través de figuras como la conexidad con derechos fundamentales. Dentro de esas manifestaciones se encuentra el coleo, un ejercicio cuyo elemento fundamental es el sometimiento de bovinos a través de maniobras que llevan a derribarlo, siendo por ello tildada como una expresión que no tiene en cuenta la protección especial a los animales dada por el ordenamiento colombiano; aún con ello, se ha arraigado en el oriente colombiano y el Estado ha tenido infructíferos intentos sobre la reglamentación de este como una práctica cultural y deportiva. 


\section{METODOLOGÍA}

La presente investigación desarrollará dos enfoques, el primero de ellos la hermenéutica de Ricoeur, y el segundo el test de culturalidad de Duarte. El primero como método para la interpretación de la realidad, textos, cuasitextos y acciones del hombre, bajo el presupuesto de que, al mezclar la narración de los textos, la interpretación se hace transversal a ellos. ${ }^{1}$ El método hermenéutico de las ciencias sociales se ha establecido no solo como hermenéutica textual, sino abarcando una precompresión del ámbito de lo práctico, es decir, desde "una dialéctica comparable a la de la comprensión y la explicación en el ámbito de lo textual". ${ }^{2}$

La convergencia entre la legibilidad de lo textual, el texto, el cuasitexto y la inteligibilidad de la acción no son fortuitas, de ellas se pueden discernir en el mismo campo práctico rasgos que llevan a la suma de la explicación y la comprensión ${ }^{3}$. Por medio de la comprensión se trata de permitir la relación entre múltiples espacios temporales teniendo en cuenta que el texto no es estático haciendo que, al referirse a la comprensión, no solo se habla del sentido del texto o composición verbal, sino de las situaciones iniciales y finales. ${ }^{4}$ Es necesario tener en cuenta la relación existente entre el texto y el lector, y, por tanto, el mundo que abre su subjetividad a partir de la objetividad del texto. ${ }^{5}$

Ahora, en relación con el segundo enfoque, debido a las vicisitudes que giran en torno a la conceptualización de cultura y los criterios para determinar cuándo estamos frente a una manifestación, se han tomado los principios planteados en la teoría multicultural de Villoro ${ }^{6}$ y Kymlicka ${ }^{7}$ tales como tolerancia, autonomía, autenticidad, sentido, eficacia, adopción restrictiva de las formas de diversidad, limitación básica a los derechos de las culturas minoritarias, garantía de los derechos de las minorías; y,

1. Rodrigo Moreno Aponte, "Hermenéutica y ciencias sociales: a propósito del vínculo entre la interpretación de la narración de Paul Ricoeur y el enfoque de investigación biográfico-narrativo", Análisis, 49, n. ${ }^{\circ}$ 90 (Bogotá: Universidad Santo Tomás, 2017).

2. Paul Ricoeur, "La hermenéutica y el método de las ciencias sociales", Cuadernos de Filosofía Latinoamericana, 34, n. ${ }^{\circ} 109$ (2013): 64.

3. Ibíd.

4. Paul Ricoeur, Del texto a la acción. Ensayos de hermenéutica II (Ciudad de México: Fondo de Cultura Económica, 2002).

5. Moreno Aponte, "Hermenéutica y ciencias sociales: a propósito del vínculo entre la interpretación de la narración de Paul Ricoeur y el enfoque de investigación biográfico-narrativo".

6. Luis Villoro, “Aproximaciones a una ética de la cultura”, en Ética y diversidad cultural, comp. León Olivé (Ciudad de México: Editorial Instituto de Investigaciones Filosóficas / Fondo de Cultura Económica, 1993).

7. Will Kymlicka, Ciudadanía cultural. Una teoría liberal de los derechos de las minorías (Barcelona: Paidós, 1996). 
por último, el principio expresado por Duarte y Parra: ${ }^{8}$ conocimiento de la pluralidad de relaciones para la creación e implementación de un mecanismo por medio del cual se puedan aplicar los principios de culturalidad sobre una práctica determinada con el objetivo de clasificarla o no como una manifestación cultural, y acercarla a la protección a través del derecho cultural. ${ }^{9}$ Con el fin demostrar la posibilidad de evaluar la culturalidad de las manifestaciones y prácticas se ha construido el denominado test de culturalidad, herramienta que permitirá elucidar los comportamientos que mutan los linderos del derecho cultural y otros como la libertad de expresión y el libre desarrollo de la personalidad.

En este sentido, en primer lugar, se han sistematizado los principios de culturalidad en etapas o pasos en los que cada uno es el sine que non del siguiente, teniendo una etapa previa exceptuada de la cadena de condiciones en que se deberá describir la situación fáctica que se evaluará; esto quiere decir que el test es de carácter secuencial. En segundo lugar, cada paso corresponde a una premisa de validación de los principios que en él se sintetizan, y, en tercer lugar, que se deben cotejar en relación con el cumplimiento o incumplimiento respecto de la práctica sometida al test. ${ }^{10}$

Son así cuatro las características esenciales del test: es secuencial, responde ante premisas de validación, da a conocer sobre el cumplimiento o incumplimiento de principios básicos, y es un proceso de contraste. ${ }^{11}$ Con todo, el test de culturalidad (figura 1) se compone de los siguientes pasos: ${ }^{12}$

Situación fáctica. Este paso corresponde a la etapa previa al test. Su fin es describir la práctica que se pretende someter al test; de manera que el evaluador y operador pueda tener los insumos necesarios y la información para responder a cada uno de los pasos propuestos en el test.

Aplicación del test de culturalidad. En los siguientes cuatro pasos se encuentran de manera sistematizada los principios de culturalidad correspondientes los planteamientos de Villoro, ${ }^{13}$ Kymlicka ${ }^{14}$ y Duarte y Parra. ${ }^{15}$ Se debe evaluar:

8. Tirson Duarte y María Parra, "La construcción de los derechos culturales: reflexión a partir de la tauromaquia” (ponencia, X Encuentro Nacional de la Red de Grupos y Centros de Investigación Jurídica y Sociojurídica, Cúcuta, 19 de octubre de 2017).

9. Tirson Duarte, "Construcción del test de culturalidad como método para la caracterización de derechos culturales", en Actas del VI Encuentro Latinoamericano de Metodología de las Ciencias Sociales (EL$\mathrm{MeCS}$ ) (Cuenca: Universidad de Cuenca, 2018): 11.

10. Ibíd.

11. Ibíd., 12.

12. Ibíd., 11-3.

13. Villoro, "Aproximaciones a una ética de la cultura".

14. Kymlicka, Ciudadanía cultural. Una teoría liberal de los derechos de las minorías.

15. Duarte y Parra, "La construcción de los derechos culturales: reflexión a partir de la tauromaquia". 
1. Desarrollo normativo. Recuento normativo, si lo hay, sobre la práctica en el ordenamiento. En primera medida pueden ser los genéricos, es decir, los contenidos en la Constitución de cada Estado; por ejemplo, la protección a la diversidad cultural en el caso colombiano. No hay protección cultural si la Constitución y las normas de su rango no contemplan postulados en su favor.

2. Uso restrictivo de la autodeterminación. La correspondencia entre la autonomía y la tolerancia en cuanto que la primera no puede constituir un límite para la realización de la segunda; ${ }^{16}$ esto es: se debe dar observancia a que, en el ejercicio de la capacidad de autodeterminación de un grupo, esta pueda excederse y encaminarse por la disminución, no reconocimiento o eliminación de otros.

3. Concentración de las necesidades individuo-colectivas. Toda manifestación tiene un carácter dual, en ocasiones presentado como una dicotomía, en la que hay una parte que corresponde a las libertades del individuo y otra a su desarrollo en colectivo. Esto no se puede evadir. Por tanto, debe existir una correspondencia entre la autodeterminación, sus creencias, actitudes e intenciones reales manifestadas a través del comportamiento, la proyección teleológica que brinda sentido a la práctica desde su vida individual y colectiva, y, en último lugar, la práctica de los medios requeridos para garantizar tales fines; es esta última la condición instrumental.

4. Prospección sociocultural. Finalmente, teniendo en cuenta la diversidad como fundamento del Estado democrático, se debe determinar si la manifestación en su esencia y sentido reconoce las minorías y mayorías en su entorno; porque justamente el derecho cultural es el diálogo entre ellas sin pretender igualdad, solo respeto, reconocimiento y garantía de ejercicio de unas y otras; además, en esa multiculturalidad es posible que se permee por otras manifestaciones por la transustanciación de valores.

Como efecto de estos planteamientos, y en virtud de la metodología planteada, el artículo se desarrollará en tres apartados. En una primera instancia una aproximación a la noción de cultura como una categoría iusfilosófica que permita el entendimiento del derecho cultural. Luego, a través de los parámetros o criterios para determinar las condiciones necesarias de existencia de una práctica cultural, el coleo sea sometido al test de culturalidad.

16. Vanessa Tassara, El multiculturalismo liberal de Will Kymlicka, Pontificia Universidad Católica del Perú, accedido 20 de febrero de 2018. 


\section{Figura 1. Test de culturalidad}

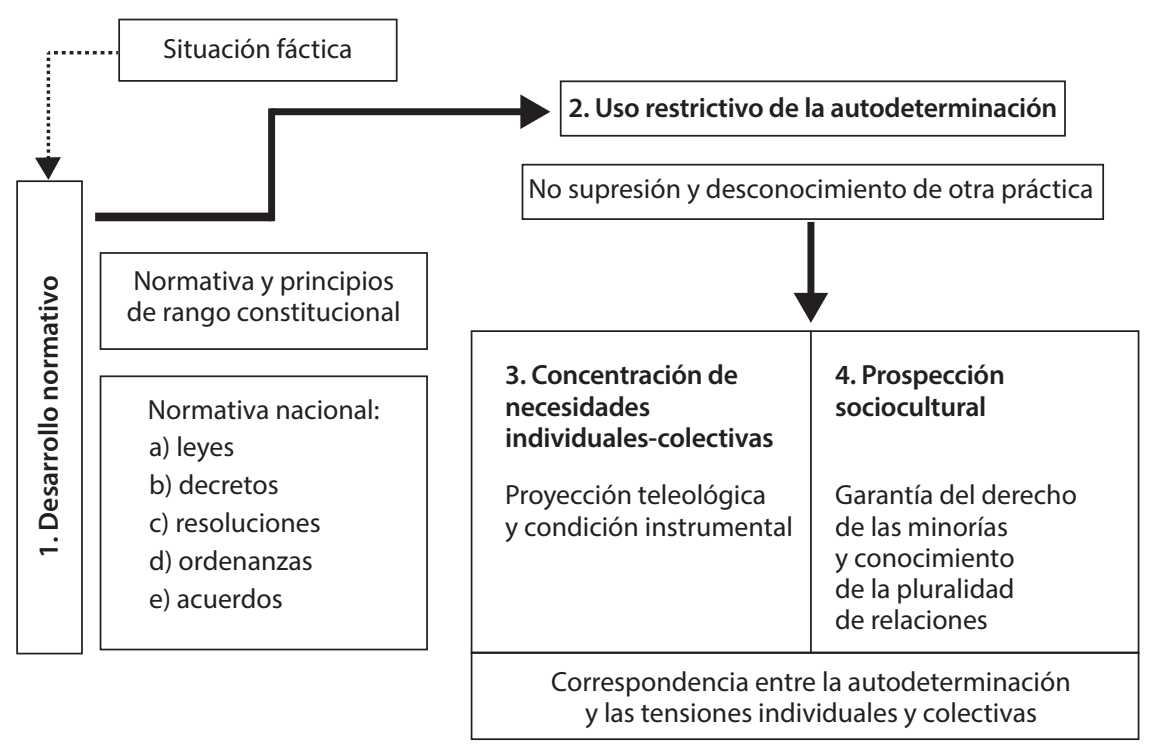

Fuente: Tirson Duarte, "Construcción del test de culturalidad como método para la caracterización de derechos culturales", en Actas del VI ELMeCS (Cuenca: Universidad de Cuenca, 2018), 11.

Elaboración: propia.

\section{DERECHO CULTURAL Y LA NOCIÓN DE CULTURA}

Para la protección de un derecho es necesario comprender lo que este abarca, y las implicaciones que tiene su garantía. Empero, el derecho cultural carece de una definición propia en el ordenamiento colombiano; de la misma manera sucede con la noción de cultura. La Corte Constitucional de Colombia ha procurado dilucidar la sombra que se cierne sobre este asunto; en primer lugar, ha considerado el derecho cultural como uno que se subsume dentro de otros, lo que se puede entender como una característica más del derecho a la libre expresión. ${ }^{17}$ Tal característica tiene fundamento en el principio de diversidad e integridad personal, a través del cual se busca que "cada individuo desarrolle su identidad se basa en las diferencias étnicas y culturales concre-

17. Colombia, Corte Constitucional, "Sentencia", C-1192, 22 de noviembre de 2005. 
tas de la nación, y no de conformidad al concepto abstracto y general de ciudadanía". ${ }^{18}$ En palabras de la Corte Constitucional:

El principio de diversidad e integridad personal no es simplemente una declaración retórica, sino que constituye una proyección, en el plano jurídico, del carácter democrático, participativo y pluralista de la república colombiana y obedece a "la aceptación de la alteridad ligada a la aceptación de la multiplicidad de formas de vida y sistemas de comprensión del mundo diferentes de los de la cultura occidental. ${ }^{19}$ (las cursivas son mías)

Aunado a ello, Gaviria ha planteado que el postulado de la protección y el reconocimiento de la diversidad cultural consagrado en la Constitución Política de Colombia presenta dos dificultades respecto al intérprete de él: conlleva un alto grado de indeterminación e implica la necesidad de ponderación con otros principios contenidos en la Carta, debido a la naturaleza conflictiva del principio en cuestión. ${ }^{20}$ La Comisión Nacional de Derechos Humanos de México-CNDH ha dicho que la transversalidad del concepto de cultura hace que esta disponga de un gran campo semántico en el que se engloban: formas de vida, literatura, lenguas, música, comunicación no verbal, creencias, ritos, ceremonias, métodos de producción, artes, costumbres y tradiciones. ${ }^{21}$

De acuerdo con ello, existen dos condiciones al hablar de cultura: una subjetiva y otra objetiva. ${ }^{22}$ En cuanto a la primera se puede explicar de la siguiente forma:

la conciencia que tienen los miembros de su especificidad, es decir, de su propia individualidad a la vez que de su diferenciación de otros grupos humanos, y el deseo consciente, en mayor o menor grado, de pertenecer a él, es decir, seguir siendo lo que son y han sido hasta el presente. ${ }^{23}$

Por su parte, la segunda condición -la objetiva- hacer referencia a los elementos materiales que distinguen al grupo. ${ }^{24}$ Encuentra Gaviria que, debido a estos condicionantes y en vista de los procesos de pluriculturalismo y la supervivencia cultural, ${ }^{25}$ nace como regla para el intérprete la maximización de la autonomía y la

18. Duarte y Parra, "La construcción de los derechos culturales: reflexión a partir de la tauromaquia".

19. Colombia, Corte Constitucional, "Sentencia", SU-510, 18 de septiembre de 1998.

20. Carlos Gaviria, "Autonomía jurisdiccional de las comunidades indígenas. Pluriculturalismo y derechos humanos", en Sentencias. Herejías constitucionales, comp. Carlos Gaviria (Colombia: Fondo de Cultura Económica, 2002).

21. México, Comisión Nacional de Derechos Humanos, Derechos humanos culturales, 2017.

22. Gaviria, "Autonomía jurisdiccional de las comunidades indígenas. Pluriculturalismo y derechos humanos".

23. José de Obieta, El derecho humano a la autodeterminación de los pueblos (Madrid: Tecnos, 1989 ), 43.

24. Ibíd.

25. El derecho a la supervivencia cultural encuentra su fundamento en el derecho a la libre autodeterminación de los pueblos, que es reconocido en el artículo 9. ${ }^{\circ}$ de la Carta Política y en el artículo $1 .^{\circ}$ del Pacto de Derechos Civiles y Políticos de 1966. 
minimización de restricciones indispensables para salvaguardar intereses de superior jerarquía. ${ }^{26}$

Se abre una posibilidad para hablar sobre la protección de aquella multiplicidad; y es esta misma Corporación que en la sentencia T-652 de 1998 hace mención de lo que se considera cultura en el ordenamiento colombiano: es el "conjunto de creaciones, instituciones y comportamientos colectivos de un grupo humano, [esto es], el sistema de valores que caracteriza a una colectividad". ${ }^{27}$ Ante la vacilación de la doctrina respecto al carácter individual o colectivo de las manifestaciones culturales, la misma Corporación ha expuesto que: "Las manifestaciones culturales no son una expresión directa de la Constitución, sino una interacción de distintos actores sociales determinados por un tiempo y espacio específicos". ${ }^{28}$

Frente a la ambivalencia en la concepción de las manifestaciones culturales algunos autores han planteado que estas nacen, principalmente, desde el individuo; otros lo hacen desde el desarrollo del individuo inmerso en colectivos. Se hace menester abordar esta disquisición teniendo en cuenta la concepción de "lo propio" atendiendo como lo principal en relación con la singularización y distinción frente a otras manifestaciones. ${ }^{29} \mathrm{Se}$ da una tendencia hacia la unificación de las culturas que, frecuentemente, es acompañada de una reacción por afirmas valores necesarios de las particularidades culturales.

Por otra parte, se han planteado dos situaciones constitutivas de la cultura: la pluralidad de individuos que reflejan diversos deseos, valores, intereses, preferencias, etc., y la imposibilidad que existe entre el aislamiento individual, es decir, la necesidad de una vida en sociedad; estas son dos situaciones que conllevan a la conformación de nuevas culturas. ${ }^{30}$ Respecto a la imposibilidad de aislamiento, Duarte y Parra han establecido el "derecho a la identidad cultural", e incluye un dejo de dignidad que no consiste en otra cosa sino en la sociabilidad humana cuando esta se manifiesta en el reconocimiento del otro, asumiendo la imposibilidad de alcanzar en solitario el desarrollo moral de la persona y también de aceptar la "igual dignidad" de todas las personas. ${ }^{31}$ Lo anterior toma en cuenta la sociabilidad como "las formas racionales que ad-

26. Gaviria, "Autonomía jurisdiccional de las comunidades indígenas. Pluriculturalismo y derechos humanos".

27. Colombia, Corte Constitucional, "Sentencia", T-652, 10 de noviembre de 1998. S/P.

28. Colombia, Corte Constitucional, "Sentencia", C-041, 1 de febrero de 2017. S/P.

29. Villoro, "Aproximaciones a una ética de la cultura".

30. Jaime Fisher, "Multiculturalismo y Ciudadanía”, Revista de Filosofía Factótum (Universidad Veracruzana), n. ${ }^{\circ} 6$ (2009).

31. Duarte y Parra, "La construcción de los derechos culturales: reflexión a partir de la tauromaquia". 
quiere nuestra sociabilidad, y las formas de comunicación que llevan a la cultura, que es el ámbito racional de nuestros conocimientos y de nuestras expresiones estéticas". ${ }^{32}$

Se entrevé la importancia de la "identidad cultural", pues es justamente el angular para cimentar el entendimiento de la noción de cultura. Según lo establecido por Salazar Benítez, es fundamental entender la identidad cultural como condición sine qua non de las manifestaciones culturales y la cultura en sí; pues es esta-desde la perspectiva individualista- el elemento fundamental para el desarrollo del ser, el eje que configura la autonomía y las condiciones de su propio bienestar. ${ }^{33}$ Desde la perspectiva colectiva Jameson trata la categoría de cultura como un encuentro entre grupos que se ha dado a lo largo de la historia; así:

se trata de un espejismo objetivo que surge de una relación entre, por lo menos, dos grupos. Es decir que ningún grupo "tiene" una cultura solo por sí mismo: la cultura es el nimbo que recibe un grupo cuando entra en contacto con otro y lo observa. Es la objetivación de todo lo que es ajeno y extraño en el grupo de contacto. ${ }^{34}$

Es evidente, desde esta perspectiva, la exclusión del carácter individual y la acepción que da a la noción de cultura como grupal respecto al proceso dialéctico del hombre en sociedad. Esta posición es concomitante con lo expuesto por la CNDH al mencionar que la cultura define la condición del género humano; pues es a través de ella que el hombre ha tenido la posibilidad de explicar su alrededor y su rol en el mundo, de allí que "cobre especial relevancia para la realización de las condiciones de existencia tanto del individuo como de las sociedades". ${ }^{35}$

No obstante, la cultura es aquella categoría considerada como un estadio individual-colectivo, y no una concepción que tiene su origen en uno o en el otro. ${ }^{36}$ Según este planteamiento, cuando se habla del sujeto como individuo inmerso en una forma de vida particular: familia, comunidad social, trabajo, academia, pero que tiene como objetivo esencial afirmarse como un "autónomo", rompe los vínculos como su comunidad "orgánica primordial", es decir, tal sujeto debe: "reconocer la sustancia de su

32. Fisher, "Multiculturalismo y Ciudadanía", 194.

33. Octavio Salazar Benítez, "El derecho a la identidad cultural como elemento esencial de una ciudadanía compleja", Revista de Estudios Políticos, n. ${ }^{\circ} 127$ (2005).

34. Fredric Jameson, "Sobre los 'Estudios Culturales'”, en Fredric Jameson y Slavoj Žižek, Estudios culturales. Reflexiones sobre el multiculturalismo (Buenos Aires: Paidós, 1993), 101.

35. México, Comisión Nacional de Derechos Humanos, Derechos humanos culturales, 4.

36. Slavoj Žižek, "Multiculturalismo, o la lógica cultural del capitalismo multinacional”, en Fredric Jameson y Slavoj Žižek, Estudios culturales. Reflexiones sobre el multiculturalismo (Buenos Aires: Paidós, 1993). 
ser en otra comunidad [...], que es a un tiempo universal y 'artificial', no 'espontánea' sino "mediada", sostenida por la actividad de sujetos libres independientes". 37

Dado ello se encuentran diversas relaciones-encuentros a través de los cuales se moldea la identidad del sujeto, como lo son comunidad local versus nación, la profesión versus la relación personal entre aprendiz y artesano, el conocimiento de la comunidad académica versus la sabiduría tradicional transmitida de generación en generación. ${ }^{38}$ De esta manera:

la identificación primaria universal comienza a funcionar como una identificación secundaria universal, a través de la cual los "valores culturales" se transustancian dando lugar a la construcción de una nueva manifestación cultural; y que, para efectos de este estudio, se encuentra desprotegida y no-reconocida por los ordenamientos. ${ }^{39}$

En palabras de Malinowski, lo anterior corresponde a las reacciones producidas entre los elementos colectivos en pequeños grupos que actúan como unidades de organización social en proceso de condicionamiento. ${ }^{40}$ Se advierte que la cultura es un conocimiento socialmente compartido, ${ }^{41}$ puesto que el hombre no es un ser cultural más que en su relación con los otros; lo que resalta el origen colectivo de la noción misma de cultura planteada líneas atrás. De modo que, de la misma forma planteada por Žižek, Enguix establece que la consideración de la cultura como proceso, como práctica y como acción social se origina en la relación con los otros, en situaciones concretas, y en un contexto dado. ${ }^{42}$

Por otro lado, y en un sentido similar, se halla que, desde la teoría cultural planteada por Geertz, esta solo se debe reducir al ámbito de los hechos simbólicos, ${ }^{43}$ pues no se le atribuye pautas a un comportamiento, entendido este como un modelo a seguir, sino que debe entenderse esta como una "pauta de significados"; esto es, que "constituyen una dimensión analítica de los comportamientos (porque lo simbólico no constituye un mundo aparte, sino una dimensión inherente a todas las prácticas)". ${ }^{44}$ En todo caso, se puede deducir que:

37. Ibíd., 165.

38. Ibíd.

39. Duarte y Parra, "La construcción de los derechos culturales: reflexión a partir de la tauromaquia".

40. Bronislaw Malinowski, "La cultura”, en Encyclopedia of Social Sciences, 1931, 4.

41. Begonya Enguix, "Cultura, culturas y antropología", Paper de investigación, 2012.

42. Ibíd.

43. Clifford Geertz, La interpretación de las culturas (Ciudad de México: Gedisa, 1987).

44. Gilberto Giménez, "La cultura como identidad y la identidad como cultura", Revista Ñ (2008): 4. 
la cultura no debe entenderse nunca como un repertorio homogéneo, estático e inmodificable de significados. Por el contrario, puede tener a la vez "zonas de estabilidad y persistencia" y "zonas de movilidad" y cambio. Algunos de sus sectores pueden estar sometidos a fuerzas centrípetas que le confieran mayor solidez, vigor y vitalidad, mientras que otros sectores pueden obedecer a tendencias centrífugas que los tornan, por ejemplo, más cambiantes y poco estables en las personas, inmotivados, contextualmente limitados y muy poco compartidos por la gente dentro de una sociedad. ${ }^{45}$

Con este planteamiento se instituye que la persona humana es un ser de carácter "singular" y "plural", debido a que: el primero permite a cada uno asumirse como diferente, único e irrepetible; al mismo tiempo que se da el segundo, que hace referencia al reconocimiento del ser humano como miembro de una sociedad y una especie donde comparte un marco común. ${ }^{46} \mathrm{Al}$ hablar de cultura se hace referencia al resultado dialéctico de las variadas relaciones universales de individuos autónomos que se consideran únicos e irrepetibles, pero que son conscientes de su convivencia e influencia de la comunidad en la que se encuentra inmerso.

Se ha establecido que son los individuos, únicamente, los que pueden dar una justificación de cualquier forma de organización social, de cualquier ente colectivo; ${ }^{47}$ se sostiene así que el individualismo moderno "sostiene que lo colectivo es una construcción de los individuos, no es algo 'natural', sino 'artificial'. Los fenómenos sociales se basan en ficciones y los fenómenos individuales se basan en hechos" ${ }^{48}$

En torno a estos se ha originado el planteamiento que la existencia de grupos y colectivos son un hecho consubstancial a la sociedad moderna y al desarrollo efectivo de los derechos de los individuos. ${ }^{49}$ Se hace evidente que la identidad se ha convertido en "un principio para organizar la sociedad en su conjunto". 50

Desde esta óptica, no se debe tratar de concebir la cultura como una creación propia de los individuos y del desarrollo independiente de cada uno, o de las relaciones grupales independientemente considerados. Se ha establecido que la cultura es el re-

\footnotetext{
45. Ibíd., 3 .

46. Sergio Tobón, Formación basada en competencias. Pensamiento complejo, diseño curricular y didáctica (Bogotá: Ecoe, 2004).

47. Nicolás López, "Sobre los derechos colectivos", en Una discusión sobre los derechos colectivos, coord. Francisco Ansuátegui (Madrid: Instituto de Derechos Humanos Bartolomé de las Casas, Universidad Carlos III de Madrid / Dykinson, 2001).

48. Ibíd., 17.

49. Gurutz Jáuregui, "Derechos individuales versus derechos colectivos: una realidad inescindible", en Una discusión sobre los derechos colectivos, coord. Francisco Ansuátegui (Madrid: Instituto de Derechos Humanos Bartolomé de las Casas, Universidad Carlos III de Madrid / Dykinson, 2001).

50. Michael Keating, Naciones contra el Estado: el nacionalismo de Cataluña, Quebec y Escocia (Barcelona: Ariel, 1996), 20.
} 
sultado del proceso dialéctico de las diversas relaciones universales de sujetos autónomos, que conviven en sociedad, y buscan que se genere a partir de la interacción con el entorno: su emancipación, para que este se forme con la pretensión de ser libre e independiente, en relación con la preocupación misma del sentido de su existencia, sin llegar a desconocer en ningún momento la pluralidad en las relaciones que lo constituyen. ${ }^{51}$ Reafirmando esto, Giménez en su interpretación de los planteamientos de Geertz ha establecido:

las identidades se construyen precisamente a partir de la apropiación, por parte de los actores sociales, de determinados repertorios culturales considerados simultáneamente como diferenciadores (hacia afuera) y definidores de la propia unidad y especificidad (hacia adentro). Es decir, la identidad no es más que la cultura interiorizada por los sujetos, considerada bajo el ángulo de su función diferenciadora y contrastiva en relación con otros sujetos. $^{52}$

Es imperativo hallar un lugar para este tipo de manifestaciones en los ordenamientos jurídicos modernos, pues, como se ha observado, estas son de vital importancia para el desarrollo del hombre mismo. De allí que los Estados deban propender por la protección y garantía a través del reconocimiento de derechos, para el caso concreto el derecho cultural, teniendo en cuenta el componente multiculturalista y pluralista del ordenamiento, y, en definitiva, el carácter de diversidad y protección de ella que se da en el modelo hegemónico, entendido como un mecanismo ideológico. ${ }^{53} \mathrm{El}$ hombre como un ser indiscutiblemente atado a lugares y personas que juegan un rol importante en su enculturación, incorporación y mentalización; es decir, se encuentra en una red que conecta y determina sus acciones. ${ }^{54}$ En este sentido:

Todos los elementos de la cultura, si esta concepción es cierta, deben estar funcionando, ser activos y eficaces [...]. El tratamiento de los rasgos culturales por atomización o aislamiento se considera estéril, porque la significación de la cultura consiste en la relación entre sus elementos, y no se admite la existencia de complejos culturales fortuitos o accidentales. ${ }^{55}$

51. Slavoj Žižek, En defensa de la intolerancia (Madrid: Sequitur, 2008); Duarte y Parra, "La construcción de los derechos culturales: reflexión a partir de la tauromaquia"; y Tirson Duarte, "Fundamentalidad [autónoma] del derecho cultural: revisión desde su núcleo esencial" (ponencia presentada en el III Congreso Internacional de Investigación, Neiva, 22 de agosto de 2019).

52. Giménez, "La cultura como identidad y la identidad como cultura".

53. Žižek, En defensa de la intolerancia.

54. Arturo Escobar, Una minga para el postdesarrollo: lugar, medio ambiente y movimientos sociales en las transformaciones globales (Lima: Universidad Nacional Mayor de San Marcos, 2010).

55. Malinowski, "La cultura", 8. 
Se debe resaltar que si la cultura es un bien que beneficia individualmente a cada sujeto, su origen se da en el colectivo y las relaciones orgánicas de los sujetos que se encuentran en él inmersos. Esto deriva del planteamiento que este tipo de manifestaciones y las prerrogativas ofrecidas por el Estado respecto a ellas no se pueden ver evocados a una titularidad exclusivamente individual, ${ }^{56}$ sino que solo puede verse asegurado su "disfrute individual mediante la colaboración y la solidaridad colectivas". ${ }^{57}$

A pesar de ello, conviene establecer una acepción para la noción de cultura, y además para lo que se debe entender como derecho cultural, para lograr un entendimiento sobre lo que es cobijado o no por la protección y garantía del Estado.

Ha sido Kymlicka quien ha expuesto que el término "multiculturalismo" abarca formas muy diferentes de pluralismo cultural. ${ }^{58}$ Se puede decir que existen diversas maneras mediante las cuales las minorías se incorporan a las comunidades políticas, desde la conquista y la colonización de sociedades que anteriormente gozaban de autogobierno hasta la inmigración voluntaria de individuos y familias. Se da la connotación desde este planteamiento que es una categoría eminentemente proteccionista y garantista respecto de las minorías; no obstante, estas diferencias en la forma de incorporación afectan a la naturaleza de los grupos minoritarios y el tipo de relaciones que estos desean con la sociedad de la que forman parte. ${ }^{59}$

Esta connotación relacionista entre multiculturalismo y minorías abarca una actitud que trata todas y cada una de las culturas locales de la manera en que el colonizador suele tratar a sus colonizados: "autóctonos" cuyas costumbres hay que conocer y "respetar". ${ }^{60}$ Aparece el multiculturalismo como una forma inconfesa de racismo que mantiene distancias, y, por lo tanto, respeta la identidad del Otro, y la concibe como auténtica y cerrada en sí misma. El respeto multicultural por la especificidad ajena a "lo propio", es decir, del Otro, es la afirmación de la superioridad propia. ${ }^{61}$

$\mathrm{Al}$ exponer posiciones contradictorias sobre el multiculturalismo, quienes se resisten afirman que este encapsula a las minorías, impidiendo su relación y transustanciación con el resto de la sociedad; $y$, en sentido contrario, quienes son partidarios de

56. Ana Garriga, “¿Son los derechos sociales derechos colectivos? La titularidad de los derechos colectivos”, en Una discusión sobre los derechos colectivos, coord. Francisco Ansuátegui (Madrid: Instituto de Derechos Humanos Bartolomé de las Casas, Universidad Carlos III de Madrid / Dykinson, 2001).

57. Francisco Contreras, Derechos sociales: teoría e ideología (Madrid: Tecnos, 1994).

58. Kymlicka, Ciudadanía cultural. Una teoría liberal de los derechos de las minorías, 10.

59. Duarte y Parra, "La construcción de los derechos culturales: reflexión a partir de la tauromaquia".

60. Žižek, En defensa de la intolerancia.

61. Ibíd. 
él, afirman que la preocupación por dicha integración es un reflejo del imperialismo cultural. ${ }^{62}$ En relación con lo planteado, se puede exponer que:

el individuo y su participación en grupo son quienes resaltan, y el argumento de igualdad que todos manifiestan para acceder a todos los beneficios que el Estado otorga -en cuanto a protección se refiere-, $\mathrm{y}$, por ello, se le empieza a dar un valor a la diversidad cultural, en función de la concesión de derechos, y las "ventajas" que esto produce. ${ }^{63}$

En tanto, una aproximación al concepto de cultura implica que se llegue a la comprensión de las creencias, prácticas y comportamientos del ser humano -entendiendo su participación colectiva para el disfrute de sus derechos-, los cuales se generan a partir de su interacción con el entorno, buscando la independencia y autonomía del sujeto, sin desconocer la pluralidad en las relaciones (orgánicas) que construyen el tejido en que se ve subsumido y lo que consigo trae. ${ }^{64}$

Una vez comprendida la noción de cultura conviene abordar la segunda, la de derecho cultural. Son Duarte y Parra quienes establecen la importancia de resaltarlo como el mediador entre las relaciones de las mayorías y las minorías ${ }^{65}$ Lo que corresponde, en un Estado democrático, a que "las relaciones entre la cultura mayoritaria y las culturas minoritarias deberían regirse por el diálogo, la negociación pacífica y el consenso (como sucede en las relaciones internacionales), nunca por la fuerza". ${ }^{66}$

Es posible que se suscite un choque entre grupos que no compartiesen sus principios básicos y su objeto cultural, y al mismo tiempo se presente la imposibilidad de persuadirse el uno al otro para que los individuos del Otro se vinculen a las prácticas propias; esta posición es lo que Žižek ha llamado transustanciación de valores. ${ }^{67}$

En relación con ello, se establece que el diálogo para detener tales conflictos respecto de diferencias en los procesos de culturización y transustanciación de una cultura en otra, se debe dar origen a la protección de la cultura bajo el derecho cultural. ${ }^{68}$ Diálogo que no debe declarar una igualdad entre desiguales pues no es suficiente para erradicar la marcada discriminación propuesta por modelos hegemónicos ${ }^{69}$ pues los argumentos basados en la igualdad solo aprueban derechos especiales para las mi-

62. Duarte, "Construcción del test de culturalidad como método para la caracterización de derechos culturales".

63. Duarte y Parra, "La construcción de los derechos culturales: reflexión a partir de la tauromaquia".

64. Ibíd. Ver también Duarte, "Construcción del test de culturalidad como método para la caracterización de derechos culturales".

65. Ibíd.

66. Tassara, El multiculturalismo liberal de Will Kymlicka.

67. Žižek, "Multiculturalismo, o la lógica cultural del capitalismo multinacional".

68. Duarte y Parra, "La construcción de los derechos culturales: reflexión a partir de la tauromaquia".

69. Žižek, En defensa de la intolerancia. 
norías si realmente se da alguna clase de desventaja relacionada con la pertenencia cultural, "Por consiguiente, el alcance legítimo de estos derechos variará según las circunstancias". ${ }^{70}$

\section{EVALUACIÓN DE LAS CONDICIONES NECESARIAS DE RECONOCIMIENTO DEL COLEO EN COLOMBIA}

El coleo tiene un fuerte arraigo en la idiosincrasia llanera de Colombia. Durante el siglo XIX, el aumento en la ganadería en países de América, especialmente en Colombia y Venezuela, dio origen a que los trabajadores idearan formas para controlar a los animales en las grandes llanuras por las que transitaban. Fue así, en la práctica del quehacer diario de la vaquería, que surgió la maniobra del coleo como un intento por atajar y someter a los bovinos que se escapaban de sus manadas, la cual consistía en tomarlos por la cola y derribarlos. ${ }^{71}$ Con el paso del tiempo, tal espectáculo fue adquiriendo mayor valor cultural, y, por lo tanto, empezó a celebrarse en las festividades de los municipios. Entonces "Las primeras manifestaciones del coleo en el departamento del Meta se llevaron a cabo en el municipio de San Martín, pero poco a poco se extendieron por todo el departamento y, hoy en día, el espectáculo tiene lugar en toda la región de la Orinoquia". ${ }^{72}$

Poco a poco, tras el disfrute colectivo, los habitantes pasaron de calles convertidas en improvisadas mangas durante sus fiestas patronales a escenarios más adecuados para su práctica y a una transformación de la misma faena. Hoy la infraestructura de las mangas contempla tribunas techadas con graderías en cemento, palcos especiales, sistemas de riego para la arena, iluminación artificial y sonido interno. La evolución en las reglas como consecuencia del paso del coleo a ser deporte federado dio origen a un reglamento amplio que ha unificado la normatividad para su práctica. ${ }^{73}$

Para los niños del Meta, entender que el caballo es su mejor amigo es algo esencial durante su crecimiento, y no solo por su utilidad, sino también por la compañía leal que este le proporciona al montador durante las largas jornadas a la deriva en la extensa llanura. ${ }^{74}$ Por eso, bañarlo, herrarlo y alimentarlo de manera adecuada son trascendentales para suministrar un buen cuidado al equino.

70. Kymlicka, Ciudadanía cultural. Una teoría liberal de los derechos de las minorías.

71. El Tiempo, "El coleo, con tradición y evolución en el llano", El Tiempo, 1 de julio de 2005.

72. Ibíd., párr. 6 .

73. Ibíd.

74. Sebastián Ríos, "El arte del coleo”, El Espectador, 5 de julio de 2017. 
En este deporte autóctono, los llaneros no tienen competencia. Según la Federación Colombiana de Coleo, no importa en qué posición se encuentre el caballista; en Colombia y Latinoamérica, no hay quien esté cerca de derribar un animal de 300 kilos o más con tanto desparpajo. El talento de estos montadores es natural en cualquier modalidad, desde las coleadas a una mano, a media silla, a medio estribo, hasta la muy compleja guesiada (técnica del coleo). ${ }^{75}$

Cuenta la historia que ni siquiera los españoles, que durante la conquista prohibieron a los mestizos del llano colombiano andar a caballo, pudieron evitar que se juntaran estas dos potencias, que se ocuparon de darle inicio a una revolucionaria invención de la cual, después, serían casi imposibles de destronar. ${ }^{76}$ La organización de este deporte tiene definidos en nuestro país torneos nacionales en categorías de promoción (menores de 9 años), preinfantil, infantil, prejuvenil, juvenil, mayores y veteranos.

\section{APLICACIÓN DEL TEST DE CULTURALIDAD}

\section{Desarrollo normativo}

A lo largo del articulado de la Constitución Política de Colombia se encuentran diversas normas que hacen referencia a la protección de a la cultura, la riqueza derivada y la diversidad. Se hallan los artículos $8^{\circ}, 9^{\circ}$, y $10^{\circ}$ que establecen la obligación en cabeza del Estado y las personas para proteger la riqueza cultural y natural de la nación, así como la soberanía nacional, en el respeto a la autodeterminación de los pueblos. Concordante con estos artículos, el artículo 70 aparece como uno de los principales fundamentos de la cultura al exponer:

Artículo 70. El Estado tiene el deber de promover y fomentar el acceso a la cultura de todos los colombianos en igualdad de oportunidades, por medio de la educación permanente y la enseñanza científica, técnica, artística y profesional en todas las etapas del proceso de creación de la identidad nacional.

La cultura en sus diversas manifestaciones es fundamento de la nacionalidad. El Estado reconoce la igualdad y dignidad de todas las que conviven en el país. El Estado promoverá la investigación, la ciencia, el desarrollo y la difusión de los valores culturales de la $\mathrm{Na}$ ción. (las cursivas son mías)

75. Ibíd.

76. Ibíd. 
El artículo 71 establece que los planes de desarrollo económico y social incluirán el fomento a la cultura; también que el Estado deberá crear incentivos para personas e instituciones que desarrollen y fomenten las manifestaciones culturales. El artículo 72 expone que el patrimonio cultural de la Nación se encuentra bajo la protección del Estado, y el patrimonio arqueológico y los bienes culturales que conforman la identidad nacional pertenecen a la nación y son inalienables, inembargables e imprescriptibles. Por último, se encuentra el artículo 15 del PIDESC, vía bloque de constitucionalidad, contiene el derecho de toda persona a participar en la vida cultural y el ejercicio del derecho con miras a la conservación, el desarrollo y la difusión de la cultura.

En cuanto a la legislación que ha adoptado la nación diferentes normas, en primer lugar, y en sentido general, la Ley 397 de 1997 señala que cultura es el conjunto de "rasgos distintivos, espirituales, materiales, intelectuales y emocionales que caracterizan a los grupos humanos y que comprende, más allá de las artes y las letras, modos de vida, derechos humanos, sistemas de valores, tradiciones y creencias". En segundo lugar, respecto a la normatividad relacionada con la práctica sometida, se encuentra el artículo $7^{\circ}$ de la Ley 84 de 1984 que exceptúa actividades como "el rejoneo, coleo, las corridas de toros, las novilladas, corralejas, becerradas y tientas así, como las riñas de gallos y los procedimientos utilizados en estos espectáculos" de la protección ofrecida por el Estatuto Nacional de Protección de los Animales.

Íntimamente relacionado con el artículo que antecede, se observa el parágrafo $3^{\circ}$ del artículo 339B contenido en el artículo 5 de la Ley 1774 de 2016 como adición al Código Penal donde se excluyen las actividades del artículo $7^{\circ}$ de la mencionada norma como circunstancias de agravación punitiva de los delitos contra la vida, la integridad física y emocional de los animales; parágrafo que a su vez fue declarado inexequible por la Corte Constitucional de Colombia en sentencia C-041 de 2017. Por último, en el año 2017 fue presentado en el Senado de la República un proyecto de ley mediante el cual se adoptaría el Reglamento Nacional del Coleo que, entre otras cosas, se consideraría:

Artículo 1. Objeto. La presente ley tiene por objeto reglamentar la preparación, organización y desarrollo de las actividades relacionadas con el deporte del coleo, reconociendo su carácter de manifestación cultural autóctona de la región de la Orinoquia. (las cursivas son mías)

No obstante, aun con la importancia de tal reconocimiento por parte de la ley colombiana, el proyecto fue retirado. En 1998, el Comité Olímpico Colombiano (COC) le reconoció su estatus de deporte, a pesar de no existir una Federación Internacional y de no desarrollarse en más de 70 países, como en principio lo exigen las normas en la materia, pues el Comité Olímpico Internacional permite aceptar nuevas disciplinas siempre y cuando se demuestre su carácter de autóctonas. En el año 2000, la Federa- 
ción Colombiana de Coleo, Fedecoleo, inicia actividades como comité provisional; en ese año Coldeportes otorgó reconocimiento deportivo al coleo mediante Resolución n..$^{\circ} 2380$ del 30 de noviembre de 2000 , y reafirmó dicha declaratoria a través de la Resolución n. ${ }^{\circ} 3100$ del 28 de diciembre de 2015.77

Actualmente, Fedecoleo tiene sede en Villavicencio y está conformada por las ligas de Casanare, Meta, Cundinamarca, Vichada, Guaviare, Arauca y la Liga Militar, y el reglamento nacional se estableció formalmente a comienzos de 1999. Se observa entonces que se ha procurado establecer la normatividad suficiente, más no necesaria, para regular la práctica.

\section{USO RESTRICTIVO DE LA AUTODETERMINACIÓN}

En el presente caso la manifestación no carece de autonomía puesto que tanto practicantes como el Estado, a través de sus instituciones, se han encargado de organizarla de tal manera que posee una estructura por medio de la cual se han determinado las maneras y formas de ejecutar sus prácticas; ello por cuanto la evolución del coleo a ser deporte federado dio origen a un reglamento amplio y que ha unificado la normatividad para su práctica. ${ }^{78}$

Ante la correspondencia entre la autonomía y la tolerancia, se halla que el Estado ha permitido el maltrato animal en la realización de esta práctica, pues desde la Ley 84 de 1989 fue excluida de los ámbitos de protección, permitiendo de esta manera que se permeara legalmente el objeto cultural de otras posibles manifestaciones. Contrario a lo contenido en la mencionada ley, se encuentra que, en la práctica, quienes hacen parte de tal manifestación no someten a los animales a cargas más allá de las que puedan soportar; por lo que, "tumbar" al ganado, animales de 300 kilos no conlleva maltrato. Se advierte que hay un uso restrictivo de la autodeterminación: en el funcionamiento de las instituciones se ha procurado en sus prácticas mantener a los animales que se ven vinculados en ellas exentos de cualquier clase de maltrato; plasmado de esta manera en el Proyecto de Ley 53 de 2017.

77. Maritza Martínez, Exposición de motivos proyecto de Ley 53 de 2017 "por medio de la cual se adopta el Reglamento Nacional del Coleo” (Colombia: Congreso de la República de Colombia, 2017).

78. El Tiempo, "El coleo, con tradición y evolución en el llano". 


\section{CONCENTRACIÓN DE NECESIDADES INDIVIDUO-COLECTIVAS}

Se encuentran justificadas la autenticidad, el sentido y la eficiencia de esta manifestación, pues, al concentrarlas buscando el sentido individual-colectivo propio de la cultura, se termina cumpliendo la condición instrumental en el entendido de que el funcionamiento de las instituciones se ha encaminado a la satisfacción de los requerimientos necesarios para garantizar los fines de la manifestación. En segundo lugar, se da la proyección teleológica en relación con el comportamiento de los practicantes, ya que se han asimilado las actitudes e intenciones como aquellas que dan sentido a la manifestación misma, y que brinda el disfrute tanto individual como colectivo en la región del Orinoco en Colombia, y brindando sentido al promocionarse la práctica desde los infantes, siguiendo ellos este estilo de vida.

\section{Prospección sociocultural}

Las garantías constitucionales, así como las demás normas producidas, no demuestran que se dé un desconocimiento de los derechos de las minorías. Sucede de diferente manera si nos enfrentamos al conocimiento de la pluralidad de relaciones, toda vez que, como se expresó anteriormente, se permea el objeto cultural de otras posibles manifestaciones, como las animalistas. Empero, la misma práctica y las manifestaciones de la Corte Constitucional han procurado zanjar tal diferencia, aboliendo el maltrato animal dentro de los procesos, toda vez que se deben proteger todos los bienes jurídico-constitucionales como la cultura y la protección de la fauna. Se da la justificación a la prospección cultural.

\section{CONCLUSIONES}

Este texto ha pretendido ser una respuesta y solución ante la incertidumbre que se cierne jurídicamente sobre la categoría cultura y sobre los criterios que se deben tener en cuenta para reconocer cuándo estamos frente a manifestaciones culturales. Respecto a las inquietudes que motivaron la creación del test de culturalidad como la noción de cultura, derecho cultural y el comprender lo que implica el reconocer una manifestación cultural, se ha logrado esclarecer un poco el camino. Como todo método es necesario seguir probando, seguir discutiendo hasta alcanzar lo más cercano a la idoneidad.

Con todo, se pretende que el test no sea tratado de manera marginal dentro de la teoría cultural, pues si bien la sistematización de los principios no es algo que se hubiese realizado en el medio para determinar prerrogativas en favor de, sí es un elemento que no solo puede ser usado desde la doctrina, sino que está planteado como uno que pueda 
Figura 2. Test de culturalidad: el coleo

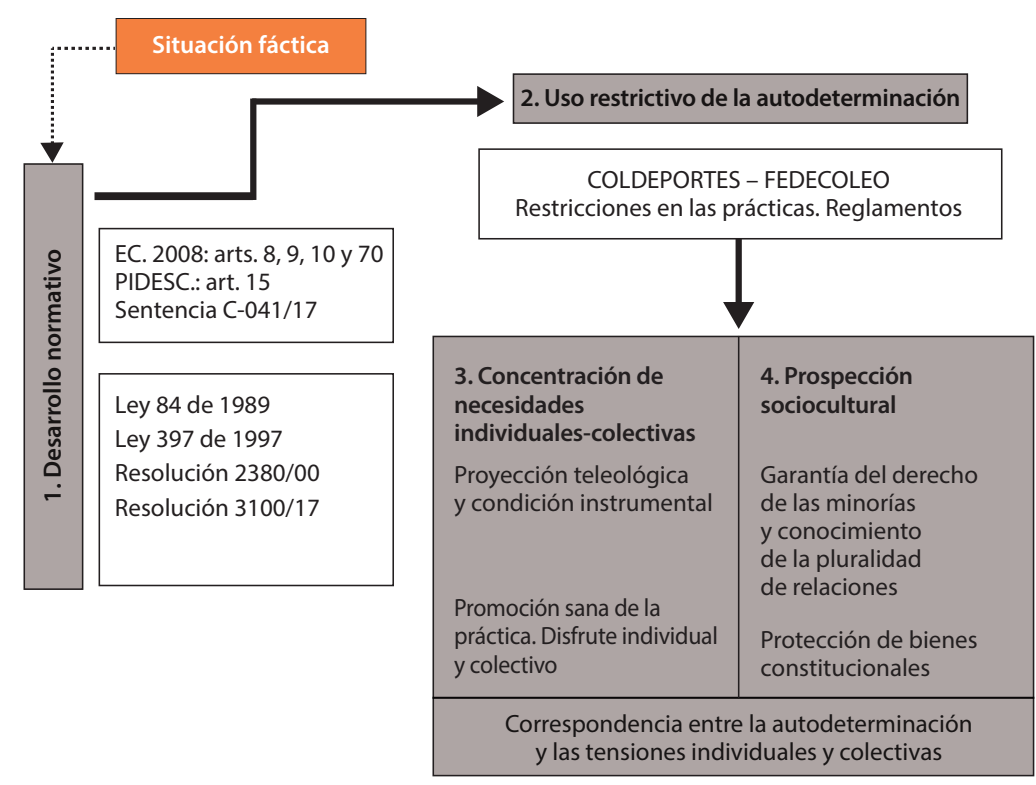

Fuente: Tirson Duarte, "Construcción del test de culturalidad como método para la caracterización de derechos culturales", en Actas del VI ELMeCS (Cuenca: Universidad de Cuenca, 2018), 11.

Elaboración: propia.

ser aplicado por operadores jurídicos con el fin de resolver las controversias suscitadas en cuanto al amparo de las manifestaciones culturales haciendo uso de los mecanismos ofrecidos por cada sistema para la garantía, interpretación, y protección.

Realizar una aproximación a la noción de cultura implica la comprensión de las creencias, prácticas y comportamientos del ser humano, desde su participación colectiva para la realización de sus derechos, los cuales se generan a partir de su interacción con el entorno, buscando la independencia y la autonomía del sujeto, sin desconocer la pluralidad en las relaciones (orgánicas) que construyen el tejido en que se ve subsumido y lo que consigo trae; por lo tanto, que el derecho deba asumir cada vez más un mayor rol en cuanto a la protección de la cultura. De allí que las entidades estatales encargadas tanto de la producción normativa como de su aplicación deban colaborar en la identificación y protección de las diversas manifestaciones, sin que el Estado mismo interfiera en las prácticas mismas, sino que lo haga más como un ente regulador en cuanto a los límites de cada manifestación respecto de otras, como se observó en el caso del coleo. 
En el conocimiento de la pluralidad de relaciones se encuentra la limitación al ejercicio del coleo, en el entendido que se da un uso restrictivo de la autodeterminación en relación con otras prácticas, puesto que ha procurado mantener a los animales que se ven vinculados en ella exentos de cualquier clase de maltrato. Lo anterior es plasmado de tal manera en el Proyecto Ley 53 de 2017. Por lo demás, una justificación frente a la autenticidad, el sentido y la eficiencia de la manifestación se encuentra que, al concentrarla buscando un sentido individual-colectivo propio de la cultura, se cumple la condición instrumental, toda vez que el funcionamiento de las instituciones se ha encaminado a la satisfacción de los requerimientos necesarios para garantizar los fines de la manifestación y la protección de otras manifestaciones y bienes jurídicos tutelados como la fauna. De allí que se pueda determinar que en función del test de culturalidad el coleo pueda ser considerado como una manifestación cultural.

\section{BIBLIOGRAFÍA}

Colombia. Corte Constitucional. "Sentencia". SU-510. 18 de septiembre de 1998.

—. "Sentencia". T-652. 10 de noviembre de 1998.

-. "Sentencia". C-1192. 22 de noviembre de 2005.

—. "Sentencia". C-666. 30 de agosto de 2010.

—. "Sentencia". C-449. 16 de julio de 2015.

-. "Sentencia". C-041. 1 de febrero de 2017.

Colombia. Estatuto Nacional de Protección de los Animales y se crean unas contravenciones y se regula lo referente a su procedimiento y competencia. Ley 84 de 1989, Diario Oficial n. ${ }^{\circ} 39.120$.

—.Ley de Maltrato Animal. Ley 1774 de 2016, Diario Oficial n. ${ }^{\circ} 49.747$.

-. Ley de Patrimonio Cultural, Fomentos y Estímulos a la Cultura, y se crea el Ministerio de Cultura. Ley 397 de 1997, Diario Oficial n. ${ }^{\circ} 43.102$.

-.Reglamento Nacional del Coleo y se dictan otras disposiciones. Proyecto de Ley 53 de 2017. https://app.vlex.com/\#vid/691326825.

Contreras, Daniela. "Penalización del maltrato animal en Colombia". Tesis de pregrado, Universidad Católica de Colombia, 2017.

Contreras, Francisco. Derechos sociales: teoría e ideología. Madrid: Tecnos, 1994.

De Obieta, José. El derecho humano a la autodeterminación de los pueblos. Madrid: Tecnos, 1989.

Duarte, Tirson. "Construcción del test de culturalidad como método para la caracterización de derechos culturales". En Actas del VI Encuentro Latinoamericano de Metodología de las Ciencias Sociales (ELMeCS) (2018): 1-21. http://elmecs.fahce.unlp.edu.ar/vi-elmecs/ actas/Duarte.pdf. 
—. "Fundamentalidad [autónoma] del derecho cultural: revisión desde su núcleo esencial". Ponencia presentada en el III Congreso Internacional de Investigación, Neiva, 22 de agosto de 2019.

Duarte, Tirson, y María Parra. "La construcción de los derechos culturales: reflexión a partir de la tauromaquia”. Ponencia presentada en el X Encuentro Nacional de la Red de Grupos y Centros de Investigación Jurídica y Sociojurídica, Cúcuta, 19 de octubre de 2017.

El Tiempo. "El coleo, con tradición y evolución en el llano". El Tiempo. 1 de julio de 2005.

Enguix, Begonya. "Cultura, culturas y antropología”. Paper de investigación. 2012.

Escobar, Arturo. Una minga para el postdesarrollo: lugar, medio ambiente y movimientos sociales en las transformaciones globales. Universidad Nacional Mayor de San Marcos. Lima. 2010. https://www.unc.edu/ aescobar/text/esp/escobar.2010.UnaMinga.pdf.

Fisher, Jaime. "Multiculturalismo y Ciudadanía". Revista de Filosofía Factótum, n. ${ }^{\circ}$ (2009): 34-45. Universidad Veracruzana. http://www.revistafactotum.com/revista/f_6/articulos/ Factotum_6_3_Jaime_Fisher.pdf.

Garriga, Ana. “Son los derechos sociales derechos colectivos? La titularidad de los derechos colectivos". En Una discusión sobre los derechos colectivos, coordinado por Carlos Ansuátegui. Instituto de Derechos Humanos Bartolomé de las Casas, Universidad Carlos III de Madrid / Dykinson, 2001.

Gaviria, Carlos. "Autonomía jurisdiccional de las comunidades indígenas. Pluriculturalismo y derechos humanos". En Sentencias. Herejías constitucionales, compilado por Carlos Gaviria. Bogotá: Fondo de Cultura Económica, 2002.

Geertz, Clifford. La interpretación de las culturas. Ciudad de México: Gedisa, 1987.

Giménez, Gilberto. "La cultura como identidad y la identidad como cultura". Revista N. 2008. https://perio.unlp.edu.ar/teorias2/textos/articulos/gimenez.pdf.

Jameson, Fredric. "Sobre los 'Estudios Culturales"”. En Fredric Jameson y Slavoj Žižek, Estudios culturales. Reflexiones sobre el multiculturalismo. Buenos Aires: Paidós, 1993.

Jáuregui, Gurutz. "Derechos individuales versus derechos colectivos: una realidad inescindible". En Una discusión sobre los derechos colectivos, coordinado por Francisco Ansuátegui. Instituto de Derechos Humanos Bartolomé de las Casas, Universidad Carlos III de Madrid / Dykinson, 2001.

Keating, Michael. Naciones contra el Estado: el nacionalismo de Cataluña, Quebec y Escocia. Barcelona: Ariel, 1996.

Kymlicka, Will. Ciudadanía cultural. Una teoría liberal de los derechos de las minorías. Barcelona: Paidós, 1996.

López, Nicolás. "Sobre los derechos colectivos". En Una discusión sobre los derechos colectivos, coordinado por Francisco Ansuátegui. Instituto de Derechos Humanos Bartolomé de las Casas, Universidad Carlos III de Madrid / Dykinson, 2001.

Malinowski, Bronislaw. "La cultura”. En Encyclopedia of Social Sciences. 1931.

Martínez, Maritza. Exposición de motivos proyecto de Ley 53 de 2017 " por medio de la cual se adopta el Reglamento Nacional del Coleo”. Congreso de la República de Colombia. 2017. 
México. Comisión Nacional de Derechos Humanos. Derechos humanos culturales. 2017. https://biblio.juridicas.unam.mx/bjv/detalle-libro/5179-derechos-humanos-culturales.

Moreno Aponte, Rodrigo. "Hermenéutica y ciencias sociales: a propósito del vínculo entre la interpretación de la narración de Paul Ricoeur y el enfoque de investigación biográficonarrativo". Análisis (Universidad Santo Tomás), 49, n. ${ }^{\circ} 90$ (2017).

Oliveros, Stephanie. "La transmutación implícita en la justiciabilidad de los derechos por la Corte Constitucional colombiana: análisis de los derechos a la consulta previa y al ambiente sano". Tesis maestría, Universidad de San Buenaventura, Cali, Colombia, 2018. http://bibliotecadigital.usb.edu.co/bitstream/10819/5714/1/Transmutaci\%C3\%B3n Impl\%C3\%ADcita_Justiciabilidad_Oliveros_2018.pdf.

ONU. Asamblea General. Pacto Internacional de Derechos Económicos, Sociales y Culturales. 16 de diciembre de 1966. Resolución 2200 A (XXI).

Ricoeur, Paul. Del texto a la acción. Ensayos de hermenéutica II. Ciudad de México: Fondo de Cultura Económica, 2002.

—. "La hermenéutica y el método de las ciencias sociales". Cuadernos de Filosofia Latinoamericana 34, n. ${ }^{\circ} 109$ (2013). https://dialnet.unirioja.es/descarga/articulo/5679959.pdf.

Riechmann, Jorge. Todos los animales somos hermanos. Ensayos sobre el lugar de los animales en las sociedades industrializadas. Madrid: Catarata, 2005.

Ríos, Sebastián. "El arte del coleo”. El Espectador. 5 de julio de 2017.

Rúa Serna, Juan. "Liberar a un ruiseñor: una teoría de los derechos para los animales desde el enfoque abolicionista". Opinión Jurídica (Universidad de Medellín): 15, n. ${ }^{\circ}$ 13: 206-25. http://www.scielo.org.co/pdf/ojum/v15n30/1692-2530-ojum-15-30-00205.pdf.

Salazar Benítez, Octavio. "El derecho a la identidad cultural como elemento esencial de una ciudadanía compleja”. Revista de Estudios Políticos, n. ${ }^{\circ} 127$ (2005): 297-322. https://dialnet.unirioja.es/descarga/articulo/1255737.pdf.

Tassara, Vanessa. El multiculturalismo liberal de Will Kymlicka. Pontificia Universidad Católica del Perú. Accedido 20 de febrero de 2018. http://iusfilosofiamundolatino.ua.es/download/El\%20multiculturalismo\%20liberal\%20de \%20\%20Will\%20Kymlicka\%20VTZ.pdf.

Tobón, Sergio. Formación basada en competencias. Pensamiento complejo, diseño curricular y didáctica. Bogotá: Ecoe, 2004.

Villoro, Luis. “Aproximaciones a una ética de la cultura”. En Ética y diversidad cultural compilado por León Olivé. Ciudad de México: Instituto de Investigaciones Filosóficas / Fondo de Cultura Económica, 1993.

Žižek, Slavoj. En defensa de la intolerancia. Madrid: Sequitur, 2008.

—. "Multiculturalismo, o la lógica cultural del capitalismo multinacional". En Fredric Jameson y Slavoj Žižek, Estudios culturales. Reflexiones sobre el multiculturalismo. Buenos Aires: Paidós, 1993. 